\title{
Adult female acne: Clinical and therapeutic particularities (Review)
}

\author{
DACIANA ELENA BRANISTEANU ${ }^{1 *}$, MIHAELA PAULA TOADER $^{2 *}$, ELENA ANDRESE PORUMB $^{1 *}$, \\ IONELA LACRAMIOARA SERBAN ${ }^{3}$, ALIN CONSTANTIN PINZARIU ${ }^{3}$, CATALINA IOANA BRANISTEANU ${ }^{4}$, \\ ANASTASIA VICOVAN $^{5}$, ANDREEA DIMITRIU ${ }^{6}$, ISABELA-ALEXANDRA FARTUSNIC ${ }^{5}$, DANIEL BODA ${ }^{7}$, \\ DANIEL CONSTANTIN BRANISTEANU ${ }^{8}$, ILARIE BRIHAN ${ }^{9}$ and ALIN CODRUT NICOLESCU ${ }^{10}$
}

Departments of ${ }^{1}$ Dermatology, ${ }^{2}$ Oral Dermatology, ${ }^{3}$ Physiology and ${ }^{4}$ Faculty of Medicine,

'Grigore T. Popa' University of Medicine and Pharmacy, 700115 Iasi; ${ }^{5}$ Department of Dermatology,

Railway Clinical Hospital, 700506 Iasi; ${ }^{6}$ Department of Dermatology, 'Arcadia' Hospitals and Medical Centers, 700620 Iasi; ${ }^{7}$ Department of Dermatology, 'Carol Davila' University of Medicine and Pharmacy, 050474 Bucharest;

${ }^{8}$ Department of Ophthalmology, 'Grigore T. Popa' University of Medicine and Pharmacy, 700115 Iasi; ${ }^{9}$ Department of Dermatology, Dermatology Clinic, Faculty of Medicine and Pharmacy, University of Oradea, 410073 Oradea;

${ }^{10}$ Department of Dermatology, 'Roma' Medical Center for Diagnosis and Treatment, 011773 Bucharest, Romania

Received October 8, 2021; Accepted November 10, 2021

DOI: $10.3892 /$ etm.2021.11074

\begin{abstract}
Acne is a chronic inflammatory condition affecting the pilosebaceous unit that was traditionally viewed as a disease of the adolescence. However, over the past several years, an increasing number of adult women have been reported to suffer from this condition. The prevalence of adult female acne ranges between 12 and 54\%. Two clinical types can be distinguished in this population, a 'retentional' and an 'inflammatory' type, which usually tend to overlap. In terms of evolution, three main subtypes can be identified: Persistent acne, which is the most frequent subtype, late-onset acne and recurrent acne. This type of acne is mainly mild-to-moderate in severity and may be refractory to conventional treatment. The etiopathogenesis is complex and has yet to be fully elucidated. It appears to involve an interaction among genetic predisposition, hormonal factors, and chronic activation of the innate immune system overlapping with external factors, such as
\end{abstract}

Correspondence to: Dr Anastasia Vicovan, Department of Dermatology, Railway Clinical Hospital, 1 Garabet Ibraileanu Street, 700506 Iasi, Romania

E-mail: anastasiavicovan@gmail.com

Dr Ilarie Brihan, Department of Dermatology, Dermatology Clinic, Faculty of Medicine and Pharmacy, University of Oradea, 10 Piața 1 Decembrie Street, 410073 Oradea, Romania

E-mail: brihan_drm@yahoo.com

"Contributed equally

Key words: adult female acne, clinical forms, etiopathogenesis, androgens, polycystic ovary syndrome, diet, stress, topical therapies, systemic therapies daily stress, Western-type diet, use of tobacco and cosmetics. The treatment may be challenging and a holistic approach is required, with special attention to the individual needs and particularities of adult women. Both topical and systemic treatments are available, with hormonal therapies being of special value in this population. The aim of the present article was to provide up-to-date, evidence-based information on the clinical presentation, etiopathogenesis and treatment of adult female acne.

\section{Contents}

1. Introduction

2. Prevalence

3. Clinical presentation

4. Etiopathogenesis

5. Treatment of adult female acne

6. Conclusions

\section{Introduction}

Acne vulgaris is a chronic inflammatory condition of the pilosebaceous unit that affects up to $85 \%$ of adolescents (1). However, research conducted over the last years shows that an increasing number of adults, particularly adult women, suffer from this condition (2). The acne that appears in women aged $>25$ years is defined as adult female acne, and differs from the adolescent type in terms of specific clinical aspects and evolution (3). The pathophysiological differences warrant a special therapeutical approach to this emerging population of patients with acne (4).

The database Google Scholar was searched for articles on this topic written in English. The key words used for the 
literature search were as follows: Adult female acne, female acne, post-adolescent acne, treatment acne, etiopathogenesis acne and prevalence adult acne. Articles that focused on and discussed the features of adult female acne, dating up to September 2021, were selected. A total of 50 papers were retrieved, including prospective, retrospective and review articles.

The aim of this review article was to provide up-to-date information on the clinical presentation, etiopathogenesis and treatment options for adult female acne.

\section{Prevalence}

Acne is a disease frequently encountered in adult women. In different studies, the prevalence of adult female acne ranges between 12 and 54\% (4-7). Goulden et al (5) were among the first to study the prevalence of adult acne by clinical examination of the participants. They reported that clinical facial acne more frequently affects adult women compared with men (12 vs. $3 \%$, respectively). Furthermore, $82 \%$ of the participants reported that the disorder had persisted from the adolescence onwards.

After the teenage years, women are more often affected by acne compared with men (1). A survey conducted in France among 3,305 adult women (aged 25-40 years) found that $24 \%$ of women had physiological acne, while $17 \%$ had clinical facial acne, with an overall prevalence of $41 \%$. The difference between physiological and clinical acne lies with the number of lesions (either pustules or papulonodules, associated or not with comedones). Thus, physiological acne is defined as the presence of 1-2 pustules or papulonodules at the time of consultation or $<5$ lesions during the last 3 months, while clinical acne consists of $>5$ lesions at the time of consultation or during the last 3 months (6). Among women with acne lesions, $49 \%$ had sequalae such as scars and/or pigmented macules, and $97 \%$ reported self-manipulation of acne lesions (6). The prevalence generally declines with age, as reported by Perkins et al (7), with $45 \%$ of the women aged $21-30$ years suffering from acne, while this percentage decreased to $12 \%$ among women aged $41-50$ years. Adult female acne is not only frequent, but its prevalence has shown an increasing trend over the past few years, as a 5-year population-based study reported a prevalence of 1.71 per 1,000 population in 2015 and a prevalence of 2.94 per 1,000 population in 2019 (8).

\section{Clinical presentation}

Acne in adult women is generally of mild to moderate severity. Severe acne is the least frequent form, with a prevalence of $1 \%$ in 385 female patients $(3,9)$.

Traditionally, two clinical types have described, according to Preneau and Dreno (3). The 'inflammatory form' consists of papulopustules and nodules that tend to leave scars, with hyperseborrhoea being a rare finding. In the 'retentional form', the lesions are represented by non-inflammatory blackheads and microcysts, while hyperseborrhoea is always present $(2,3,10)$. In adult female acne, the lesions are mainly located on the lower part of the face, in a 'surgical mask' distribution pattern, namely the mandibular region, chin and perioral region, while they may also extend to the anterior cervical region $(2-4,11)$.
However, this typical form of acne has been disputed recently, mainly due to studies that reported other areas of involvement. A prospective observational study including 374 female patients concluded that $89.8 \%$ of the women had acne lesions that involved multiple areas of the face, such as the forehead, cheeks, mandible and temples, with a range of severity that was similar to those observed in adolescents. A mixed form of facial acne, with both inflammatory and non-inflammatory lesions, was the most common clinical presentation. By contrast, only a small subset of patients $(11.2 \%)$ had lesions confined to the mandibular region (12).

When analyzing the literature, there are three main subtypes of evolution that can be identified: i) Persistent acne is the most frequent type, identified in $70-80 \%$ of cases, in which acne is a continuum from adolescent years to adulthood $(3,9,11,13)$; ii) late-onset acne, reported in $20-40 \%$ of women, which appears for the first time after the age of 25 years $(4,9,10)$; and iii) recurrent acne, which is present in adolescence and, after a variable clearing period, it reappears and manifests as adult acne; this latter subtype is poorly described, yet it may be more common than is reported, partly because current epidemiological surveys rarely inquire if there is a recurrence of lesions following a clearing period after adolescence $(3,13)$.

\section{Etiopathogenesis}

The pathogenesis of acne in the adult female population is complex and has yet to be fully elucidated (9). Traditionally, four main pathogenic factors have been implicated in the development of acne: Increased sebaceous secretion, bacterial colonization by Cutibacterium acnes (formerly known as Propionibacterium acnes), follicular hyperkeratinization with subsequent plugging of the follicle and complex inflammatory processes that involve both acquired and innate immunity $(2,13,14)$.

Recent studies have found that the development of acne is not simply associated with hyperproliferation of $C$. acnes, but rather with a loss of balance between the different phylotypes in association with a dysbiosis of the skin microbiome $(2,15)$. The selection of a subset of $C$. acnes strains, including the phylotype $\mathrm{IA}_{1}$, as well as the loss of diversity of the other phylotypes, trigger innate immune system activation and lead to cutaneous inflammation (2). In addition, the gut microbiome, through its interactions with the skin microbiome, has been found to be involved in acne. This finding may change the direction in acne treatment to anti-inflammatory 'ecobiological' therapies (15).

Although no clear difference has yet been established between the acne that develops in adult women and the one observed in adolescents, two main pathophysiological factors appear to be particularly involved: Peripheral hormonal factors and the chronic stimulation of the innate immune system by resistant strains of $C$. acnes $(3,16)$.

Several other factors may be of special interest in adult women, such as genetic predisposition, diet, use of tobacco, chronic stress, cosmetics and medications.

Genetic factors play an undeniable role in the pathogenesis of acne in adult women. They influence the number, size and activity of the sebaceous glands, mediate the action of hormonal factors, promote follicular keratinization and 
regulate the innate immune system (2). Although acne is not a heritable condition, its incidence tends to be increased among members of the same family (17). Dréno et al (12) reported that over than half of the subjects $(56.8 \%)$ had a first-degree relative with acne, while Skroza et al (9) identified a family history of acne in $70.6 \%$ out of 385 female patients.

Hormones, particularly androgens, have a well-established role in the etiopathogenesis of acne. This is supported by four clinical observations. First, androgen-insensitive subjects, who do not have functional androgen receptors, do not produce sebum and do not develop acne; second, elevated levels of dehydroepiandrosterone sulfate (DHEA-S), a precursor of testosterone, have been associated with the early development of acne in prepubertal girls; third, the acne that is associated with disorders that are characterized by hyperandrogenism, such as polycystic ovary syndrome (PCOS), is highly responsive to hormonal treatments; and fourth, even in women with normal androgen levels, hormone-based therapies, such as oral contraceptives and anti-androgen medications, are highly effective $(14,18,19)$.

Androgens in women are derived from three major sources: The ovaries, the adrenal glands and the skin (via peripheral conversion). The pituitary luteinizing hormone and follicle-stimulating hormone stimulate the ovaries to produce small quantities of androgens, such as DHEA-S and testosterone, that are subsequently released into the circulation. DHEA-S, which can be further metabolized into the more potent androgens androstenedione and testosterone, is also produced by the adrenal glands. The skin has all the enzymes required for the synthesis of androgens from cholesterol de novo and also for converting weak androgens into strong androgens. However, most of the testosterone and dihydrotestosterone (DHT) produced by the skin are derived from circulating DHEA-S, DHEA, androstenedione and estradiol $(20,21)$. In the skin, androgen receptors are found at the highest density in the basal cells and differentiating sebocytes. They are also found in cells of the dermal papilla and outer root sheath of the hair follicle, vascular endothelial and smooth muscle cells, epidermal and follicular keratinocytes and also in the sweat glands (19,20). Sebaceous gland growth and sebum production is stimulated by testosterone, DHEA-S and DHT $(2,22)$. Not only the sebum production is stimulated by the androgens, therefore supporting $C$. acnes colonization, but sebocytes may also directly contribute to the inflammatory process of the sebaceous gland via increased production of pro-inflammatory cytokines (23).

Androgen-mediated sebum production is a necessary but not sufficient condition for the development of acne (20). Hyperandrogenism, when present, is usually associated with additional clinical signs, such as hirsutism, androgenetic alopecia, seborrhea, menstrual disorders (amenorrhea or oligomenorrhea), virilization (deepening of the voice, increased muscle mass, decreased breast size, clitoromegaly), ovulatory disfunction, infertility and metabolic syndrome $(2,19)$. Although PCOS is the most common cause of hyperandrogenism (80\% of cases), the differential diagnosis should include androgen-secreting neoplasms of the adrenal gland or ovaries, pituitary and hypothalamic tumors, late-onset non-classical congenital adrenal hyperplasia, the hyperandrogenism-insulin resistance-acanthosis nigricans syndrome, the seborrhea-acne-hirsutism-alopecia syndrome, as well as exogenous androgens (19,24).

PCOS should be diagnosed according to the revised Rotterdam criteria (2003) that define PCOS as fulfilling two of the following three criteria: Menstrual disorders (amenorrhea or oligomenorrhea); clinical and/or biochemical hyperandrogenism; and ultrasonographic documentation of ovarian volume $\geq 10 \mathrm{ml}$ (preferred criterion when using transducer frequencies $<8 \mathrm{mHz}$ ) and/or 25 follicles per ovary (criterion preferred when using transducers with frequencies $\geq 8 \mathrm{mHz}$ ) (25). According to these criteria, the presence of regular menstrual cycles does not exclude the diagnosis of PCOS $(19,26)$.

PCOS is a heterogeneous disorder in terms of phenotypes and clinical manifestations, and also in terms of metabolic consequences. The classic, most severe phenotypes include clinical and/or biochemical hyperandrogenism and oligo-ovulation, and have been found to be associated with the most severe insulin resistance and metabolic comorbidities. The ovulatory phenotype, which consists of hyperandrogenism and abnormal ovarian morphology on ultrasound, is associated with moderate insulin resistance and metabolic comorbidities. The normoandrogenic phenotype has a weak association with insulin resistance and metabolic comorbidities $(19,25)$. Due to the endocrine, reproductive, cardiovascular and oncological complications that may occur, it is important that adult women presenting with acne to the dermatologist should be investigated to exclude PCOS (27).

While some adult women have true hormonal disorders, several have hormonal levels within the normal range (13). In these patients, acne may be a manifestation of end-organ hypersensitivity due to an increase in the number and sensitivity of the androgen receptors of the sebocytes and keratinocytes to normal circulating levels of hormones, and also due to increased peripheral hormonal conversion (2). As previously mentioned, sebocytes and keratinocytes present an enzymatic system capable of locally producing testosterone and DHT. DHT is a 5- to 10-fold more potent androgen compared with its precursor, testosterone, and it is also less likely to be metabolized into estrogen by aromatase. Enzymes involved in the metabolism of androgenic hormones exhibit an abnormal activity, with increased pre-hormone peripheral conversion of DHEA-S and androstenedione into more potent forms $(2,13)$.

A number of adult female patients with acne report worsening of their condition in the premenstrual period, as well as premenopausally, during pregnancy and during the use of progestin-only contraceptives. During these periods, women experience hormone fluctuations with a relative increase in the levels of those with androgenic activity $(2,13,28)$. The reported incidence of these flare-ups slightly differs across studies. Geller et al (29) found that up to $65 \%$ of the participants described a worsening of their acne symptoms around and even during their menses. Anaba and Oaku (17) observed premenstrual flares of acne in $58.9 \%$ of the patients included in their study. They also reported that premenstrual flares do not contribute to acne severity, contrary to what has been previously reported (29).

The association between acne and diet remains unclear. Studies have suggested that a Western diet, defined as high 
calorie intake, high glycemic load, high meat and fat intake, and high consumption of cow's milk and dairy products, may be associated with acne flares in predisposed individuals $(13,28,30)$. This type of diet increases insulin and insulin-like growth factor 1 (IGF1) levels. IGF1 is a potent mitogen that induces cell proliferation and inhibits apoptosis of sebocytes and keratinocytes and stimulates sebum production (30). The gonads have receptors for both insulin and IGF1, and their action stimulates the production of androgens and also inhibits the action of aromatase that converts testosterone to estradiol. In addition, an elevated serum insulin concentration suppresses sex hormone-binding globulin (SHBG) and raises androgen levels (2). The aggravation of acne is a result of the effect of upstream activation of insulin/IGF1 signaling on metabolic regulations mediated by forkhead box protein $\mathrm{O} 1$ (FoxO1) and the nutrient-sensitive mammalian target of rapamycin complex 1 kinase. This triggers increased lipid and protein synthesis, proliferation and differentiation of keratinocytes, hyperplasia of the sebaceous gland and increased sebaceous lipogenesis $(2,30)$. Some early findings support the beneficial effects of probiotics on acne (31). In one randomized, placebo-controlled, double-blind study that involved 20 adult patients with acne comparing a supplement containing Lactobacillus rhamnosus GG to a placebo over a period of 12 weeks, patients who received the probiotic exhibited a clinical improvement in acne; furthermore, the skin biopsies that were performed revealed that, in the probiotic group, the gene expression of IGF1 in the skin was reduced by $32 \%$ and gene expression of FoxO1 was increased by $65 \%(\mathrm{P}<0.001)$. In the placebo group, no such differences were observed. Those findings indicated that supplementation with probiotics can normalize skin expression of genes involved in insulin signaling and may improve the appearance of adult acne (32).

Previous studies $(2,33)$ reported that an important percentage of adult women described that acne lesions worsened during stressful periods. Stress induces secretion of several neurotransmitters, cytokines and hormones. Corticotropin-releasing hormone (CRH), cortisol and other glucocorticoids bind to skin receptors and can aggravate acne lesions by regulating lipid synthesis in sebocytes and promoting an increase in lipid content at lower concentrations. Certain genes associated with $\mathrm{CRH}$ are expressed at higher levels in acne-affected compared with acne-free skin. In addition to CRH and cortisol, substance P (SP), a neuropeptide that is associated with stress and pain, plays an important role. SP increases immunoreactivity to IL-1, IL- 6 and TNF- $\alpha$, thereby affecting the production of inflammatory mediators. Sleep deprivation may also act as a stressor, promoting secretion of stress-related hormones of $(2,33)$.

\section{Treatment of adult female acne}

When managing a female patient with acne, a holistic approach should be used, combining standard treatments with adjunct therapy and cosmetics use, and also considering the distinctive characteristics of each individual (16,34). All the central tenets of acne management may also be applied during the treatment of adult female acne (19). However, some additional considerations should be kept in mind during the course of treatment. Women aged $\geq 25$ years tend to have higher rates of treatment failure: $\sim 82 \%$ of treatment failures involve multiple courses of systemic antibiotics and $\sim 32 \%$ occur following treatment with isotretinoin. When the recurrence of acne lesions occurs shortly after a course of isotretinoin, it should raise the suspicion of an underlying endocrinological disorder and appropriate investigation should follow $(14,19)$.

Several other factors must be taken into account when treating an adult female patient with acne, such as the extent, severity and duration of acne, response to former treatments, the high psychosocial impact of this disease, increased sensitivity of the skin with an increased likelihood of skin irritation, possibility of pregnancy, predisposition to scarring and post-inflammatory hyperpigmentation, as well as patient preferences and treatment costs $(2,4,16,35,36)$.

Topical therapies. The same topical agents that are used in the treatment of adolescent acne are also helpful in treating the adult type. Topical therapies may be used as monotherapy or in combination with other topical agents or even oral agents, in order to target all the factors implicated in the pathogenesis of acne $(34,37,38)$. The available topical agents include topical retinoids, antibiotics, benzoyl peroxide, azelaic acid and dapsone, as well as various fixed-dose combinations of these active ingredients $(2,13)$.

Topical retinoid monotherapy is recommended in adult women with mild comedonal acne, or as part of a combination regimen for moderate to severe acne $(4,13)$. The topical retinoids that have been approved by the Food and Drug Administration (FDA) are adapalene, tazarotene and tretinoin. These agents help normalize follicular keratinization and decrease keratinocyte cohesiveness and, in doing so, reduce follicular occlusion and comedone formation. They also compete with factors involved in the inflammatory response of acne, enhance penetration of other topical acne medications and accelerate resolution of acne-induced post-inflammatory hyperpigmentation (39). Their use, however, must be adjusted to the skin of older patients who may be susceptible to irritation $(4,13)$. Adapalene $0.3 \%$ gel is one of the topical retinoids that was found to be efficient and well-tolerated in the adult female population. A post hoc analysis of two phase 2 and 3 studies revealed greater efficacy of adapalene compared with vehicle in reducing total lesion count at the 12th week, and also a significant reduction of the inflammatory lesions (by 61\%) and non-inflammatory lesions (by $51 \%)(2,13,40)$.

Topical antibiotics for acne accumulate in the follicle and have been hypothesized to function through anti-inflammatory and antibacterial mechanisms. Clindamycin is the only agent currently recommended, as it is less frequently associated with bacterial resistance (41). Antibiotics are best used in combination with benzoyl peroxide, which increases efficacy and decreases the development of resistant bacterial strains (37). Due to the significant increase in $C$. acnes strains resistant to topical antibiotics, the use of these agents alone is not recommended $(2,37)$. In the light of emerging bacterial resistance, a randomized, double-blind, placebo-controlled study was conducted to evaluate the efficacy and safety of topical $2 \%$ ketoconazole cream. Ketoconazole cream, a topical antifungal with anti-inflammatory and antiandrogenic actions, can decrease the lipase activity of $C$.acnes and was shown to work against antibiotic-resistant isolates in vitro (42). The proportion of participants 
with acne improvement from baseline ( 42.9 vs. $9.5 \%, \mathrm{P}=0.015)$ and the success rate in the ketoconazole group were significantly higher than that of the placebo group (45.0 vs. $14.3 \%$, $\mathrm{P}=0.043$ ). Therefore, the authors concluded that ketoconazole monotherapy is a viable option for the treatment of mild adult female acne, with an excellent safety profile (42).

Benzoyl peroxide $(2.5,4,5,8$ and $10 \%$ gel $)$ has bactericidal, anti-inflammatory and anti-hyperkeratinization properties, and it is recommended as monotherapy for mild-to-moderate acne $(34,37,41)$. In adult women, it can induce skin irritation or dryness, and concentrations $>5 \%$ are not recommended for use in this particular population (34). A randomized, double-blind, placebo-controlled trial conducted over 12 weeks, which enrolled 63 adult women aged 24-45 years, found that the combination of topical benzoyl peroxide and low-dose spironolactone $(50 \mathrm{mg} /$ day) was effective in improving moderate adult female acne, with an acceptable side-effect profile. Therefore, the authors proposed this regimen as an option for treating moderate adult female acne (43).

Azelaic acid (20\% cream or $15 \%$ gel or foam) is recommended as first-line monotherapy for both inflammatory and non-inflammatory adult female acne. Furthermore, azelaic acid has anti-tyrosinase activity and it is suitable for the treatment of post-inflammatory hyperpigmentation. As azelaic acid is naturally found within the body, systemic side effects are unlikely to occur, making it safe for use during pregnancy and breastfeeding $(34,41)$. Azelaic acid is considered safe by the FDA and represents an important option for women of childbearing age who wish to become pregnant (2).

A novel approach to the topical treatment of acne, which was recently approved by the FDA, is clascoterone, the first topical androgen antagonist developed to treat acne in both men and women. Clascoterone binds to the androgen receptors in the sebaceous glands and hair follicles, thus inhibiting the binding of DHT, with good efficacy, safety and tolerability (23).

Systemic therapy. In moderate to severe forms of adult female acne, and even in mild forms that are associated with scarring, prolonged duration or failure to respond to topical therapies, systemic treatments are usually required (16). Oral antibiotics, isotretinoin and hormonal therapies are all commonly used to treat acne in this patient population (13).

Systemic antibiotics should not be used alone, given the increase in the incidence of antibiotic resistance. Systemic antibiotics combined with topical therapies (except topical antibiotics) may show synergistic efficacy. The addition of benzoyl peroxide prevents the occurrence of resistant strains, while retinoids can effectively target most pathogenic factors (16). Their use should be limited to a maximum of 3-4 months (41). Tetracycline and its derivates are the first choice of antibiotics, but they must be avoided during pregnancy due to the inhibition of fetal skeletal growth and dental alterations. Erythromycin, although it is associated with greater bacterial resistance, is the first choice during pregnancy and lactation $(2,16)$.

Hormonal therapies, including anti-androgens and oral contraceptive pills (OCs), are highly effective in the treatment of adult female acne, even in patients without serum hormone changes. They are particularly useful in patients with severe seborrhea, flare-ups prior to menstruation, endocrine disorders, persistent inflammatory acne that is refractory to standard treatments, late-onset acne, androgenic alopecia, and when oral contraception is needed or desired. As OCs primarily reduce sebum production, they may have to be combined with other classes of agents, such as azelaic acid, retinoids and benzoyl peroxide formulations $(2,16)$.

Androgen-receptor blockers include cyproterone acetate, spironolactone, drospirenone and flutamide. Cyproterone acetate, which is an androgen receptor inhibitor and also decreases the secretion of gonadotropins, is available in combination with ethinylestradiol and is recommended for the treatment of mild to moderate adult female acne $(2,34,44)$.

Spironolactone, an aldosterone antagonist and an inhibitor of $5 \alpha$-reductase, is recommended at low doses (50-150 $\mathrm{mg} /$ day) as monotherapy or as an adjunctive therapy to topical treatments for adult female acne that is resistant to standard treatments $(34,45,46)$. Despite not being FDA-approved for the treatment of acne, it has long been used off-label. Most recently, a retrospective study including 395 adult female patients reported promising results on the use of spironolactone for acne. The patients received a median dose of $100 \mathrm{mg}$ daily. The majority of the patients (91.4\%) exhibited an improvement with spironolactone treatment and $66.1 \%$ had a complete remission. Efficacy was observed across all severity subtypes of acne, including patients with papulopustular and nodulocystic acne. Therefore, the authors concluded that spironolactone is a safe and effective treatment for adult female acne (45). Spironolactone is efficient not only in clearing facial acne, but also truncal acne, which can be particularly difficult to treat. Over two-thirds of women in a recent retrospective study achieved complete clearance of their truncal acne (47). A randomized double-blind trial is currently undergoing in Nantes, France to demonstrate the superiority of spironolactone in terms of efficacy, in order to establish it as an alternative to cyclines (48).

OCs containing estrogen in the form of ethinylestradiol combined with a progestin are recommended for the treatment of mild to moderate adult female acne (34). There are currently four OCs approved by the FDA for the treatment of acne: Ethinylestradiol/norgestimate, ethinylestradiol/norethindrone acetate/ferrous fumarate, ethinylestradiol/drospirenone and ethinylestradiol/drospirenone/levomefolate. These pills decrease androgen production at the ovarian level and also increase SHBG levels, which binds free circulating testosterone and renders it unavailable for binding to and activating the androgen receptor. In addition, they reduce $5 \alpha$-reductase activity and block the androgen receptor (37). Before prescribing, physicians should be aware of the absolute and relative contraindications for OCs (absolute: Pregnancy, current breast cancer, breastfeeding $<6$ weeks postpartum, diabetes with end-organ damage or $>20$ years duration, age $\geq 35$ years and heavy smoking, history of or current deep vein thrombosis or pulmonary embolism, major surgery with prolonged immobilization, history or current ischemic heart disease, history of cerebrovascular accident, active viral hepatitis, severe decompensated cirrhosis and liver tumors; and relative: Breastfeeding 6 weeks- 6 months postpartum, postpartum $<21$ days, age $\geq 35$ years and light smoking, history of breast cancer with $\geq 5$ years of no disease) (37).

Isotretinoin remains a highly important non-hormonal treatment option for acne in adult women (19). It is 
recommended as a first-line treatment for severe nodular cystic acne and as second-line treatment for severe acne that is unresponsive to adequate courses of standard therapy. Although its effectiveness is undeniable, there are significant dose-related side effects, the most serious and irreversible of which is teratogenicity; therefore, it is contraindicated during pregnancy $(2,34)$.

Given the recurrent nature of this disease, maintenance therapy is required to minimize the likelihood of relapse after treatment. As first-line maintenance therapy for adult female acne, topical retinoids, such as adapalene $0.1 \%$ or tazarotene $0.1 \%$, are recommended, with azelaic acid (15 or $20 \%$ ) being a viable alternative (34).

As alternative treatments for acne, blue and red light therapy and photodynamic therapy have been recommended by certain guidelines $(41,49)$. Blue light exerts antimicrobial effects against C. acnes and also suppresses sebocyte proliferation. Red light diminishes sebum production. Although the efficacy of these treatments in reducing acne lesions has been demonstrated, there is currently a lack of evidence to support the use of these interventions and more data are required $(41,50)$.

\section{Conclusions}

Adult female acne is a distinctive entity in the acne disease spectrum, which is very common in the dermatological practice. Unfortunately, there are currently few clinical studies addressing the specificity of the disease in this population. Adult female acne differs from the adolescent variant, not only in the clinical presentation and etiopathogenesis, but also in its tendency for chronicity, which may last until the postmenopausal period. These differences require a different treatment approach that addresses the specific needs of each individual. This disease not only affects cosmetic appearance, but it can also have a great psychological impact, adversely impacting the quality of life and overall well-being of those affected. Due to these issues, adult female acne poses a major challenge in clinical practice and its etiopathogenesis warrants further investigation.

\section{Acknowledgements}

Not applicable.

\section{Funding}

Publishing funds were provided by the Association of Dermatologists from Moldova.

\section{Availability of data and materials}

Not applicable.

\section{Authors' contributions}

DEB, ACN, MPT and DCB contributed to the study design, participated in the review process and prepared the manuscript. ACP, AV, IAF and CIB contributed to collecting the relevant literature, data analysis and critical interpretation. IB, EAP, AD, DB and ILS conceived the review and modified the manuscript. All the authors have read and approved the final version of the manuscript.

\section{Ethics approval and consent to participate}

Not applicable.

\section{Patient consent for publication}

Not applicable.

\section{Competing interests}

The authors declare that they have no competing interests.

\section{Authors' information}

CIB is a medical student at University of Medicine and Pharmacy, 'Grigore T. Popa' Iasi, Romania.

\section{References}

1. Collier CN, Harper JC, Cantrell WC, Wang W, Foster KW and Elewski BE: The prevalence of acne in adults 20 years and older. J Am Acad Dermatol 58: 56-59, 2008.

2. Bagatin E, Freitas THP, Rivitti-Machado MC, Machado MCR, Ribeiro BM, Nunes S and Rocha MADD: Adult female acne: A guide to clinical practice. An Bras Dermatol 94: 62-75, 2019.

3. Preneau $\mathrm{S}$ and Dreno B: Female acne-a different subtype of teenager acne? J Eur Acad Dermatol Venereol 26: 277-282, 2012.

4. Dréno B: Treatment of adult female acne: A new challenge. J Eur Acad Dermatol Venereol 29: 14-19, 2015.

5. Goulden V, Stables GI and Cunliffe WJ: Prevalence of facial acne in adults. J Am Acad Dermatol 41: 577-580, 1999.

6. Poli F, Dreno B and Verschoore M: An epidemiological study of acne in female adults: Results of a survey conducted in France. J Eur Acad Dermatol Venereol 15: 541-545, 2001.

7. Perkins AC, Maglione J, Hillebrand GG, Miyamoto K and Kimball AB: Acne Vulgaris in Women: Prevalence across the life span. J Womens Health (Larchmt) 21: 223-230, 2011.

8. Rueda LJ, Porras A and Rico A: Prevalence of adult female acne in Colombia: A population-based study. Int J Women's Dermatol (In press).

9. Skroza N, Tolino E, Mambrin A, Zuber S, Balduzzi V, Marchesiello A, Bernardini N, Proietti I and Potenza C: Adult acne versus adolescent acne. J Clin Aesthet Dermatol 11: 21-25, 2018.

10. Holzmann R and Shakery K: Postadolescent acne in females. Skin Pharmacol Physiol 27 (Suppl 1): S3-S8, 2014.

11. Rajegowda HM, Suman BS, Basavapura Madegowda SK, Kalegowda D and Shettar Rajendra BS: A clinicoepidemiological study of adult acne among females: Is it surpassing the adolescent acne? Clin Dermatol Rev 5: 71-77, 2021.

12. Dréno B, Thiboutot D, Layton AM, Berson D, Perez M and Kang S: Large-scale international study enhances understanding of an emerging acne population: Adult females. J Eur Acad Dermatol Venereol 29: 1096-1106, 2015.

13. Zeichner JA, Baldwin HE, Cook-Bolden FE, Eichenfield LF, Friedlander SF and Rodriguez DA: Emerging issues in adult female acne. J Clin Aesthet Dermatol 10: 37-46, 2017.

14. Tan AU, Schlosser BJ and Paller AS: A review of diagnosis and treatment of acne in adult female patients. Int $\mathrm{J}$ Womens Dermatol 4: 56-71, 2018.

15. Dréno B, Dagnelie MA, Khammari A and Corvec S: The skin Microbiome: A new actor in inflammatory acne. Am J Clin Dermatol 21: 18-24, 2020.

16. Dreno B, Bagatin E, Blume-Peytavi U, Rocha M and Gollnick H: Female type of adult acne: Physiological and psychological considerations and management. J Dtsch Dermatol Ges 16: 1185-1194, 2018

17. Anaba EL and Oaku IR: Adult female acne: A cross-sectional study of diet, family history, body mass index, and premenstrual flare as risk factors and contributors to severity. Int J Womens Dermatol 7: 265-269, 2020. 
18. Uysal G, Sahin Y, Unluhizarci K, Ferahbas A, Uludag SZ, Aygen $\mathrm{E}$ and Kelestimur F: Is acne a sign of androgen excess disorder or not? Eur J Obstet Gynecol Reprod Biol 211: 21-25, 2017.

19. Kamangar $F$ and Shinkai K: Acne in the adult female patient: $A$ practical approach. Int J Dermatol 51: 1162-1174, 2012.

20. Bienenfeld A, Azarchi S, Lo Sicco K, Marchbein S, Shapiro J and Nagler AR: Androgens in women: Androgen-mediated skin disease and patient evaluation. J Am Acad Dermatol 80 1497-1506, 2019.

21. Ianosi S, Ianosi G, Neagoe D, Ionescu O, Zlatian O, Docea AO, Badiu C, Sifaki M, Tsoukalas D, Tsatsakis AM, et al: Age-dependent endocrine disorders involved in the pathogenesis of refractory acne in women. Mol Med Rep 14: 5501-5506, 2016

22. Zouboulis CC, Picardo M, Ju Q, Kurokawa I, Törócsik D, Bíró T and Schneider MR: Beyond acne: Current aspects of sebaceous gland biology and function. Rev Endocr Metab Disord 17: 319-334, 2016.

23. Kircik LH: Androgens and acne: Perspectives on clascoterone, the first topical androgen receptor antagonist. Expert Opin Pharmacother 22: 1801-1806, 2021.

24. Schmidt TH and Shinkai K: Evidence-based approach to cutaneous hyperandrogenism in women. J Am Acad Dermatol 73: 672-690, 2015

25. Escobar-Morreale HF: Polycystic ovary syndrome: Definition, aetiology, diagnosis and treatment. Nat Rev Endocrinol 14: 270-284, 2018.

26. Daghash R, Daghash A and Abu-Taha M: The clinical practice of acne treatment in polycystic ovary syndrome. Int J Pharmaceut Res: 13, 2021.

27. Begum S, Hossain MZ, Rahman MF and Banu LA: Polycystic ovarian syndrome in women with acne. J Pakistan Association Dermatol 22: 24-29, 2012.

28. Shah N, Shukla R, Chaudhari P, Patil S, Patil A, Nadkarni N and Goldust M: Prevalence of acne vulgaris and its clinico-epidemiological pattern in adult patients: Results of a prospective, observational study. J Cosmet Dermatol 20: 3672-3678, 2021.

29. Geller L, Rosen J, Frankel A and Goldenberg G: Perimenstrual flare of adult acne. J Clin Aesthet Dermatol 7: 30-34, 2014.

30. Romańska-Gocka K, Woźniak M, Kaczmarek-Skamira E and Zegarska B: The possible role of diet in the pathogenesis of adult female acne. Adv Dermatol Allergol 33: 416-420, 2016.

31. Baldwin $\mathrm{H}$ and Tan J: Effects of diet on acne and its response to treatment. Am J Clin Dermatol 22: 55-65, 2021.

32. Fabbrocini G, Bertona M, Picazo Ó, Pareja-Galeano $\mathrm{H}$, Monfrecola G and Emanuele E: Supplementation with Lactobacillus rhamnosus SP1 normalises skin expression of genes implicated in insulin signalling and improves adult acne. Benef Microbes 7: 625-630, 2016

33. Albuquerque RG, Rocha MA, Bagatin E, Tufik $S$ and Andersen ML: Could adult female acne be associated with modern life? Arch Dermatol Res 306: 683-688, 2014.

34. Dréno B, Layton A, Zouboulis CC, López-Estebaranz JL, Zalewska-Janowska A, Bagatin E, Zampeli VA, Yutskovskaya Y and Harper JC: Adult female acne: A new paradigm. J Eur Acad Dermatol Venereol 27: 1063-1070, 2013.

35. Anaba EL and Cole-Adeife MO: Cross-sectional study of quality of life impairment of adult female acne patients. Ann Clin Biomed Res 2: 135, 2021.
36. Taranu T, Constantin MM, Toader MP, Esanu I and Mocanu M: The benefits of using the iodine solution in the treatment of acne at pregnant women. Rev Chim 69: 2343-2345, 2018.

37. Zaenglein AL, Pathy AL, Schlosser BJ, Alikhan A, Baldwin HE, Berson DS, Bowe WP, Graber EM, Harper JC, Kang S, et al: Guidelines of care for the management of acne vulgaris. J Am Acad Dermatol 74: 945-973.e33, 2016.

38. Andrese E, Vata D, Porumb V, Martu C, Statescu L and Solovastru L: New complex ( $\alpha$ hydroxy acids, $\beta$ hydroxy acids, niacinamide and glycerin) for local treatment of acne vulgaris. Rev Chim 67: 1591-1593, 2016.

39. Kolli SS, Pecone D, Pona A, Cline A and Feldman SR: Topical retinoids in acne vulgaris: A systematic review. Am J Clin Dermatol 20: 345-365, 2019.

40. Berson D and Alexis A: Adapalene $0.3 \%$ for the treatment of acne in women. J Clin Aesthet Dermatol 6: 32-35, 2013.

41. Bagatin E, Rocha MADD, Freitas THP and Costa CS: Treatment challenges in adult female acne and future directions. Expert Rev Clin Pharmacol 14: 687-701, 2021.

42. Chottawornsak N, Chongpison Y, Asawanonda $\mathrm{P}$ and Kumtornrut C: Topical 2\% ketoconazole cream monotherapy significantly improves adult female acne: A double-blind, randomized placebo-controlled trial. J Dermatol 46: 1184-1189, 2019.

43. Patiyasikunt M, Chancheewa B, Asawanonda P, Noppakun N and Kumtornrut C: Efficacy and tolerability of low-dose spironolactone and topical benzoyl peroxide in adult female acne: A randomized, double-blind, placebo-controlled trial. J Dermatol 47: 1411-1416, 2020.

44. Husein-ElAhmed H: Management of acne vulgaris with hormonal therapies in adult female patients. Dermatol Ther 28: $166-172,2015$

45. Roberts EE, Nowsheen S, Davis MDP, McEvoy MT, Newman CC, Sartori Valinotti JC, Sciallis GF, Torgerson RR and Wetter DA: Treatment of acne with spironolactone: A retrospective review of 395 adult patients at Mayo Clinic, 2007-2017. J Eur Acad Dermatol Venereol 34: 2106-2110, 2020.

46. Han JJ, Faletsky A, Barbieri JS and Mostaghimi A: New acne therapies and updates on use of spironolactone and Isotretinoin: A narrative review. Dermatol Ther (Heidelb) 11: 79-91, 2021.

47. Garg V, Choi JK, James WD and Barbieri JS: Long-term use of spironolactone for acne in women: A case series of 403 patients. J Am Acad Dermatol 84: 1348-1355, 2021.

48. Poinas A, Lemoigne M, Le Naour S, Nguyen JM, Schirr-Bonnans S, Riche VP, Vrignaud F, Machet L, Claudel JP, Leccia MT, et al: FASCE, the benefit of spironolactone for treating acne in women: Study protocol for a randomized double-blind trial. Trials 21: 571, 2020.

49. Salavastru C, Tiplica GS, Branisteanu DE and Fritz K: Light-based inflammatory acne treatments. Hautarzt 69: 27-34, 2018.

50. Oon HH, Lim SYD and Goh CL: Adult acne vulgaris. In: Acne: Current Concepts and Management. Suh DH (ed). Springer International Publishing, Cham, pp35-44, 2021.

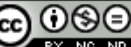

This work is licensed under a Creative Commons Attribution-NonCommercial-NoDerivatives 4.0 International (CC BY-NC-ND 4.0) License. 